Article

\title{
Gene Delivery System Using Droplet Injector and Temperature-Controlled Planarian Holder
}

\author{
Michael Lee ${ }^{1}$, Jing-Jie Syu ${ }^{2}$, Chia-Ying Chu ${ }^{2, *}$ and Yen-Wen $\mathrm{Lu}^{1,3, *(1)}$ \\ 1 Department of Bio-Industrial Mechatronics Engineering, National Taiwan University, Taipei 10617, Taiwan; \\ michaellee1120@gmail.com \\ 2 Department of Life Science, National Taiwan University, Taipei 10617, Taiwan; r06b21016@ntu.edu.tw \\ 3 Institute of Biotechnology, National Taiwan University, Taipei 10617, Taiwan \\ * Correspondence: cychu@ntu.edu.tw (C.-Y.C.); yenwenlu@ntu.edu.tw (Y.-W.L.); \\ Tel.: +886-2-3366-9876 (C.-Y.C.); +886-2-3366-5346 (Y.-W.L.)
}

Received: 26 July 2018; Accepted: 16 August 2018; Published: 21 August 2018

\begin{abstract}
A microinjection system for gene delivery to a planarian was presented with materials widely used by manufacturers. The system consists of a nanoliter droplet generator/injector and a planarian holder. Glass capillary needles were used to consistently generate droplets and to inject droplets into a planarian. The holder provides a low-temperature environment that immobilizes the planarian for injection. Our system was tested and showed successful injections of microbeads and droplets with double-stranded RNA into the planarian. The results demonstrated the capability of our system as an alternative for gene delivery for studying gene functions in planarians or other living objects for regenerative medicine studies.
\end{abstract}

Keywords: capillary; microinjection; RNA interference; gene delivery

\section{Introduction}

Planarians, well-known flatworms that can regenerate any missing part of their bodies, are perhaps one of the most important species in regenerative medicine, which holds great promise in treating physical illnesses [1-4]. Planarian regeneration requires neoblasts, undifferentiated cells that can divide and regenerate lost portions of planarians $[4,5]$. While researchers decipher the planarian regenerative mechanism, genetic tools, such as RNA interference (RNAi), in which small RNAs are employed for inhibiting translation or gene expression, are most-frequently used [6]. To perform RNAi, gene delivery is usually required. An appropriate and effective technique of gene delivery is therefore vital in the study of planarian regeneration mechanisms.

One common gene-delivery method for planarians is ingestion, whereby planarians are fed with genetic-substance-mixed food [6,7]. This method, however, is not applicable for studying the functions of the genes related to starvation conditions. In addition, it is difficult for planarians to eat the same amount of food mixed with genetic-substance every time, so the dosage of ingested DNA or RNA may not be consistent. Therefore, a reliable gene delivery method that can be applied to planarians is required.

Microinjection is another widely used gene delivery technique for organ cellular/organismal objects [8,9]. It is simple, direct, and efficient. Different formats of microinjection, including microfluidic chips or glass capillary needles, have been demonstrated to generate liquid droplets that contain biomolecules of interest in a precise manner into individual biological objects for a variety of studies [10-15]. For the microinjection in worm biology, a microfluidic device regulated by on-chip pneumatic valves has shown loading, immobilization, injection, and sorting of single Caenorhabditis elegans [15]. Another microfluidic device with an open chamber was developed to immobilize single 
C. elegans through negative pressure with a micropipette used for the microinjection into the worm body [16]. Although microfluidic chips may seem to be a promising tool in microinjection in $C$. elegans, they are not convenient while conducting microinjection on larger worms, such as a planarian whose typical size is several $\mathrm{mm}$ in width and $5 \sim 10 \mathrm{~mm}$ or more in length This is mainly due to the size matching (i.e., C. elegans has a size of $40 \mu \mathrm{m}$ in width and $1 \sim 1.5 \mathrm{~mm}$, that is at the range of the microchannel figure size).

A microinjection, which can immobilize a larger worm is therefore preferred for a planarian. The injection system must also be able to consistently generate a nanoliter droplet (or micrometer-scale droplet). Herein, we have developed such a microinjection system using simple materials, materials widely used by manufacturers. Glass capillary needles are used to generate droplets and to inject droplets into the planarian. The system also has a planarian holder to offer a low-temperature environment for freezing (or immobilizing) the planarian. This is the preferred method, compared to conventional manual immobilization using ice, which is time-consuming and can easily damage the worm due to time and less-controlled manual operations. These limitations of manual injection significantly restrict the use of the microinjection techniques. The development of our system for worm injection is highly desired.

\section{Materials and Methods}

\subsection{Droplet Generator/Injector}

A pneumatic micro injector was developed by our laboratory, providing required pressures in the generation of droplets. As shown in Figure 1A, it has five major components: a stepper motor, a linear slide, a plunger, a clamper and a needle. The stepper motor, which functions as the actuator, moves the linear slide and generates the movement required for injection. The linear slide translates the movement to the plunger, thereby pushing the liquid or substance inside the needle and creating a droplet to be sent for injection. The plunger is made from a steel rod and attached to the linear slide. The clamper is modified from a drill chuck to hold the needle. The needle is made of a pulled glass capillary.

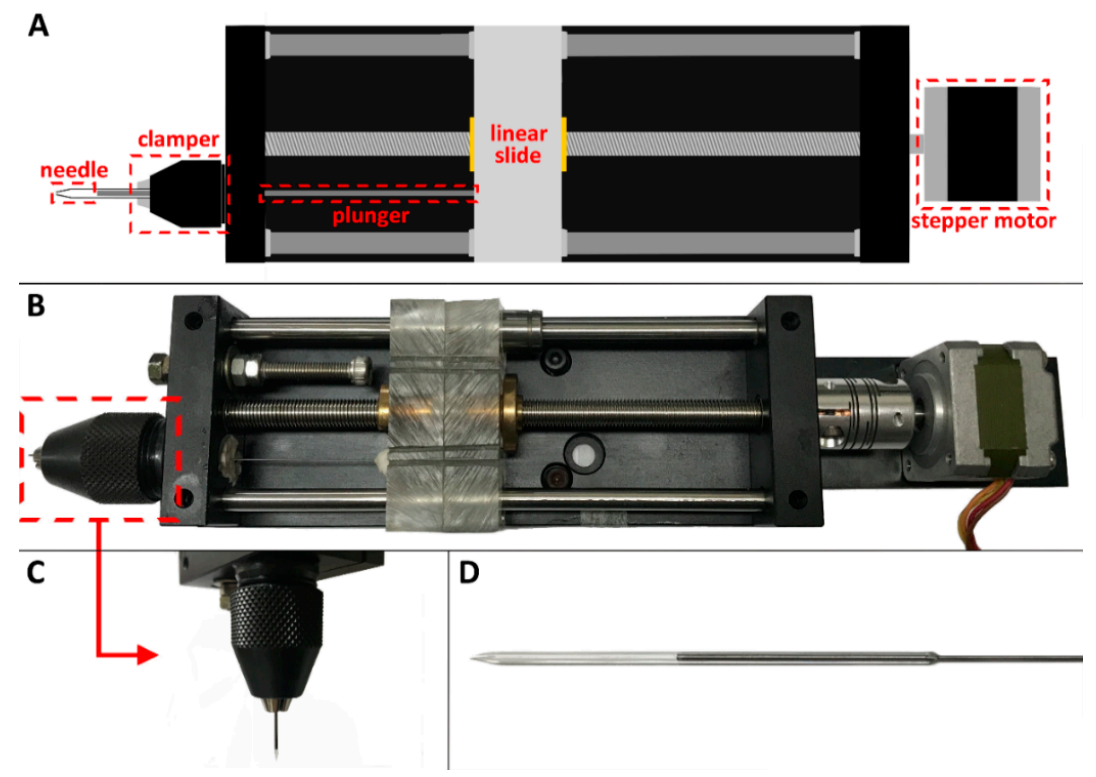

Figure 1. Our droplet generator/injector has five components: a stepper motor, a linear slide, a plunger, a clamper and a needle, in (A) schematic drawing and (B) photographic picture. (C) The clamper that is modified from a drill chuck for clamping a needle; (D) a steel rod as the plunger and a pulled glass capillary as needle and syringe. 
During the droplet generation, the stepper motor provides rotational motion with the slide translating it into linear motion. The plunger can then move linearly, thereby pushing the liquid in the plunger outward through the glass needle for generating droplets. Thus, the size of the droplet depends upon the angle in which the stepper motor rotates; the linear motion from the slide screw; and the cross-sectional area of the plunger. In other words, the droplet volume $\left(\mathrm{V}_{\text {droplet }}\right)$ can be expressed as:

$$
\mathrm{V}_{\text {droplet }} \propto\left[\left(\frac{\theta}{360^{\circ}} \times \mathrm{P}\right) \times \mathrm{A}\right]
$$

where $\theta$ is the angle, which the stepper motor rotates, $\mathrm{P}$ is the pitch of the slide screw, and $\mathrm{A}$ is the cross-sectional area of the plunger.

The smallest droplet can be generated in theory when the stepper motor rotates in its unit step angle (i.e., $\theta=1.8^{\circ}$ ). Larger droplets can be obtained when the stepper motor rotates in larger degrees. In our setup, the smallest droplet for microinjection is about $1 \mathrm{~nL}$.

In addition, the orifice size and shape of the glass needles play vital roles. The glass needles are made from glass capillaries with a micropipette puller by adjusting the temperature of a capillary (HEAT), thereby, limiting the velocity of the capillary when it is being pulled (VEL) and exerting a force to pull the capillary apart (PULL). Different combinations in HEAT, VEL, and PULL are tested to receive optimal values for a variety of orifice sizes, or inner diameter (I.D.) of the needles for generating droplets with consistent volume.

In making the glass needle, HEAT (which controls the temperature when pulling a needle) and the orifice size are slightly exponentially correlated with a coefficient of determination of 0.5573 . VEL, which limits the velocity of the needle carriage system, and taper length are log-correlated with a coefficient of determination of 0.9769 . PULL, which controls the force of the hard pull, and the needle's I.D. are negatively correlated with a coefficient of determination of 0.8982 .

In addition, to reduce the chance of needle tip breakage during the injection, the needle tip was produced with a thicker, more solid wall, thereby optimizing the pipette-pulling conditions. For example, a larger value of HEAT (i.e., the higher temperature on pulling glass) can lead to a smaller viscosity of glass. This allows the glass capillary to be pulled more easily with the tip becoming smaller and smoother. A larger value of HEAT was adapted to produce the needle tip with a clear cut. Two other parameters, including "VEL" (to regulate the tapering length) and "PULL" (to regulate the orifice size) were optimized.

Moreover, while the pulling the capillary apart, the capillary tip can produce a sharp, uneven edge. As a result, the tip may pick up debris or contaminants, thereby causing droplet volume fluctuations and the glass needle to be ineffective for microinjection. It then becomes necessary to polish the injector needles for increasing the injection success rate. A series of tests have been performed to ensure the needle tips that have even/symmetric edges without any polishing or treatment during pulling; the needles listed in Table 1, therefore, can be obtained. In particular, the needles with an inner diameter (I.D.) of $10 \mu \mathrm{m}$, which is close to the size of neoblast, are to be used in our following experiments. 
Table 1. Needles with Orifice Sizes Obtained by Adjusting Parameters (e.g., HEAT, VEL, and PULL) in Micropipette Puller.

\begin{tabular}{cccccc}
\hline HEAT & VEL & PULL & I.D. & Photos \\
\hline 617 & 10 & 10 & $10 \mu \mathrm{m}$ & \\
\hline 615 & 10 & 10 & $12 \mu \mathrm{m}$ & \\
\hline 620 & 0 & 10 & $15 \mu \mathrm{m}$ & \\
\hline 600 & 20 & 10 & $20 \mu \mathrm{m}$ & \\
\hline
\end{tabular}

\subsection{Planarian Holder}

Figure 2 showed our planarian holder. It had a platform with the temperature of the surface controlled by a feedback system, including a microcontroller, a thermoelectric cooler, and a resistance thermometer. The thermoelectric cooler was turned $\mathrm{ON}$ when the temperature was higher than the setpoint $\left(12{ }^{\circ} \mathrm{C}\right)$ and $\mathrm{OFF}$ when the temperature was below the setpoint. The temperature on the platform of the holder started around room temperature $\left(25^{\circ} \mathrm{C}\right)$ and decreased to $12.25 \pm 0.09{ }^{\circ} \mathrm{C}$ after $36.0 \mathrm{~s}$. It was not stable enough for injection because the planarians kept wiggling on the platform. Thus, a proportional-integral-derivative controller (PID controller) was added to reduce the temperature variation and to stabilize the temperature of the platform with good accuracy.

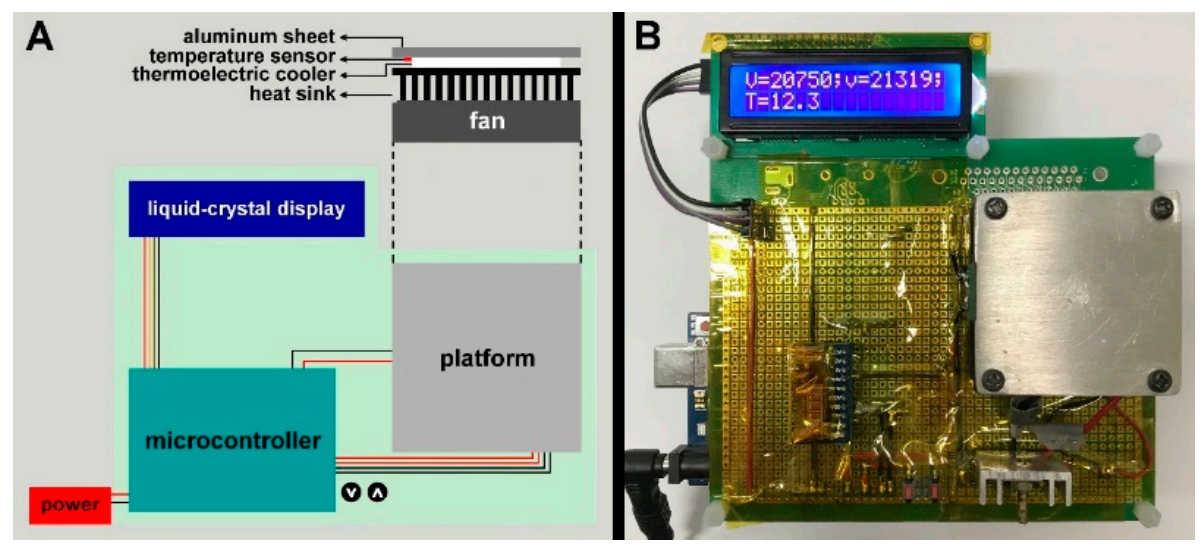

Figure 2. (A) Schematic and (B) photograph of our planarian holder. The holder has a PID controller, which can control the temperature of the platform at $12{ }^{\circ} \mathrm{C}$, to immobilize the planarian, thereby allowing the microinjection to be performed.

With the PID controller, our system had an average temperature of $12.00{ }^{\circ} \mathrm{C}$ and a standard deviation of $0.02{ }^{\circ} \mathrm{C}$. It was stable and precise enough for the experiment requirements. Figure 3 showed a planarian on the temperature-controlled platform. At first, the planarian kept wiggling on the platform at room temperature as it was out of the water (Figure 3A). As the temperature decreased, the planarian moved slower and gradually became immobilized. It was in an ideal condition for injection, as shown in Figure 3B. 


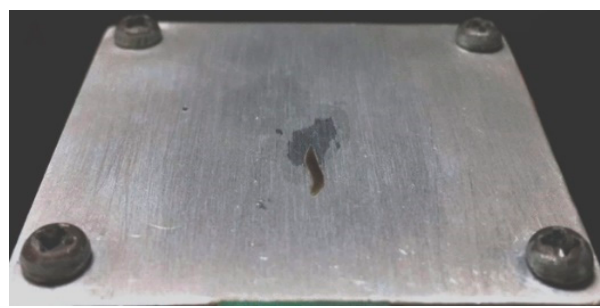

(A)

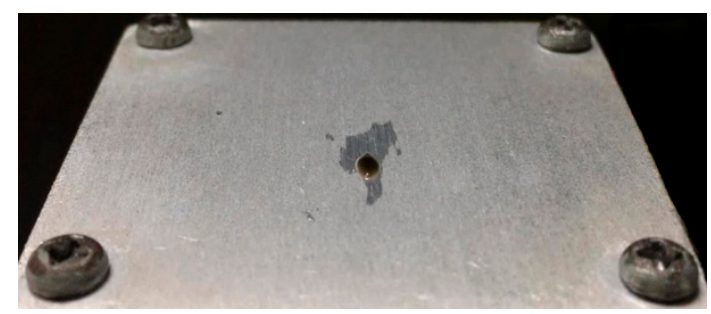

(B)

Figure 3. Temperature-controlled planarian holder. (A) The planarian kept wiggling before the platform was turned ON; (B) The planarian was immobilized (ready for injection) when the platform was turned ON.

\subsection{Characterization and Evaluation of Our Microinjection System}

The adaptability of our microinjector was verified by delivering fluorescent microbeads to the planarian. Fluorescent microbeads (BD ${ }^{\mathrm{TM}}$ Cytometer Setup and Tracking Beads, San Jose, CA, USA),

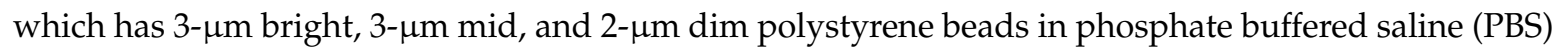
with bovine serum albumin (BSA), and sodium azide, is diluted at 1:10 with PBS and employed in our preliminary test. The droplet volume for microinjection was set to $50 \mathrm{~nL}$, which contained $\sim 9 \times 10^{9}$ fluorescent microbead, higher than the minimum dose of $10^{9}$ molecules for effective microinjection.

Figure 4 depicted the standard procedures, where our microinjection was injecting the target (e.g., microbead or genetic substances) into the planarian. A planarian was initially placed on the platform. The holder was turned $\mathrm{ON}$ with the temperature of the platform decreasing to $12{ }^{\circ} \mathrm{C}$. The planarian shrank in size and became immobilized, which was an ideal condition for injection. The microinjector was adjusted in $X Y$ directions to aim the planarian ventrally (Figure 4c), and then it was slowly adjusted downwards to make the needle tip contact with the planarian, when the gloss of the contact surface on the planarian changed. The injector was adjusted slightly downwards (about $0.02 \mathrm{~mm}$ ) to penetrate the planarian for microinjection (Figure 4d). Figure 4e showed a planarian with a glass needle penetrated. The droplet with microbead solution was injected into the planarian. 


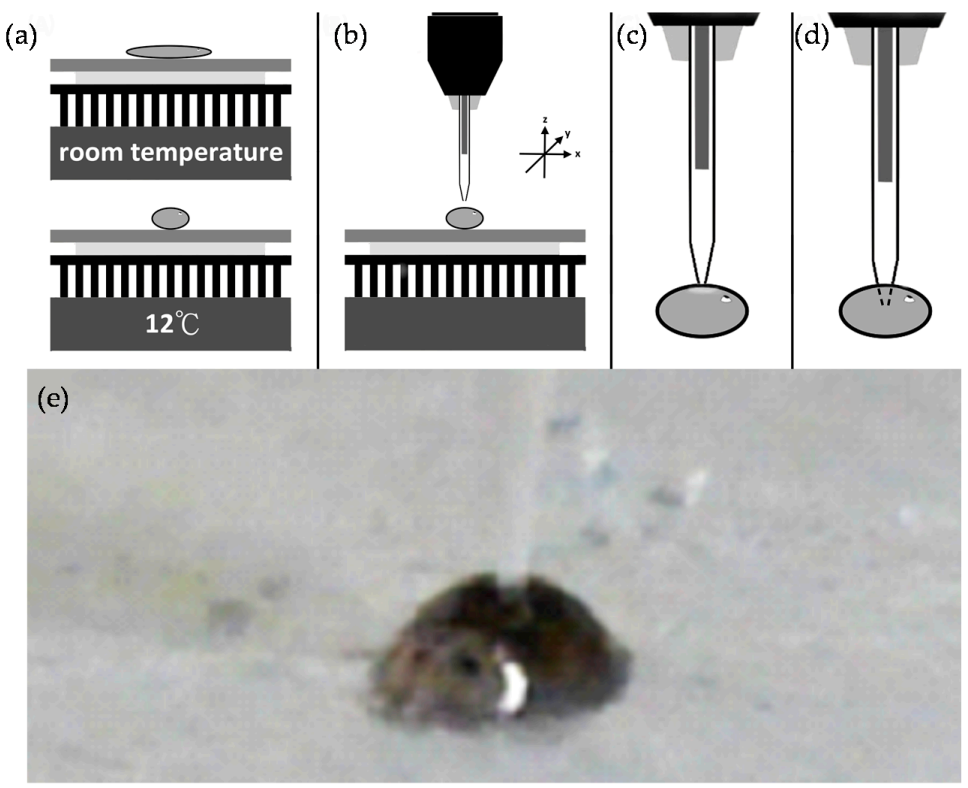

Figure 4. The procedure of the microinjection onto planarian. (a) A planarian was placed on the platform; (b) as soon as the planarian holder was turned $\mathrm{ON}$, the temperature of the platform reached $12{ }^{\circ} \mathrm{C}$ with the planarian immobilized; (c) the injection needle was carefully aligned to the target planarian; (d) the injector needle moved downwards to penetrate the planarian for microinjection; (e) a planarian with a glass needle penetrated. The droplet with microbead solution was injected into the planarian.

\subsubsection{Double-Stranded RNA (dsRNA) Preparation}

For practical applications, we used our microinjection system to deliver DjAgo2 doubled-stranded RNA (dsRNA) into planarian, and monitored the effect of RNAi induced by DjAgo2 dsRNA. DjAgo2 dsRNA and the control YFP dsRNA were synthesized as previously described with modifications [17]. In brief, the $\sim 3 \mathrm{~kb}$ DjAgo2 fragment containing $2787 \mathrm{bp}$ of CDS was identified using the sequenced and assembled Dugesia japonica transcriptome (Chu Lab, unpublished) with the use of BLAST, and cloned into RBC T\&A cloning vector after RT-PCR. The cloned DjAgo2 cDNA was then used as the template for amplification of target sequence by PCR with the following primers that harboring $\mathrm{T} 7$ promoters. After purification, the PCR product was used as the DNA template to synthesize DjAgo2 dsRNA by using MegaScript T7 kit according to manufacturer's manual. The control YFP dsRNA was synthesized by the same method with pEYFP-C1 as the template for PCR. The DjAgo2 dsRNA used for our microinjection was about 1000 bp long at $0.2 \mu \mathrm{g} / \mu \mathrm{L}$, or $1.8 \times 10^{11}$ molecules $/ \mu \mathrm{L}$.

Ago2 forward primer: 5'-GCCTAATACGAGACACTATAGAAGGTGTTTGGGATATGAGAGG-3' Ago2 reverse primer: 5'-GCCTAATACGACTCACTATAGGGTAACAATGCCGAAATTTGAT-3' YFP forward primer: 5'-GCCTAATACGAGACACTATAGGGATGGTGAGCAAGGGC-3' YFP reverse primer: 5'-GCCTAATACGAGACACTATAGGGACTTGTACAGCTCGTCC-3'

\subsubsection{Microinjection of dsRNA into Planarians}

Thirty planarians approximately $5 \mathrm{~mm}$ long were tested. Fifteen planarians were grouped as the experimental group for DjAgo2 dsRNA injection and the other fifteen planarians were used as the control group for YFP dsRNA injection, which had no significant effect on planarian.

The injection was executed every 3 days for approximately 2-3 weeks. The injection procedures were similar to the microbeads injection. The droplet volume for microinjection was set to $50 \mathrm{~nL}$, which contained approximately $9 \times 10^{9}$ molecules, higher than the minimum dose of $10^{9}$ molecules for effective microinjection. 


\title{
2.3.3. dsRNA Delivery by Feeding
}

Feeding RNAi was performed as described with modification. Thirty planarians similar in size as to those used in microinjection were fed with chicken liver mix containing $0.25 \mu \mathrm{g} / \mu \mathrm{L}$ dsRNA every 3 days. After six rounds of feeding, animals were collected for analysis of gene expression.

\subsubsection{Expression Analysis}

Nineteen days after the first injection, or after six feedings, total RNA was extracted from intact planarians survived in the experimental group (dsAgo2) or control groups (dsYFP) with TRIzol reagent. The RNA concentration was measured with a spectrometer to ensure the RNA quality for the following step in reverse transcription. The complementary DNA (cDNA) was synthesized from the RNA template via reverse transcription (RT) by using SSIII Reverse transcriptase (Invitrogen, Carlsbad, CA, USA). The synthesized cDNAs were then diluted and used for analyzing DjAgo2 and DjpiwiA expression by quantitative PCR (qPCR). The primers for RT-qPCR were listed as below:

\author{
Djß-tubulin forward: 5'-TGTCAGTGAACAATTTACTGCC-3' \\ Dj $\beta$-tubulin reverse: $5^{\prime}$-GTTGATATTCACTAACCAAATCATTC-3' \\ DjAgo 2 forward: 5'-CCTGTAATATTTCTCGGTGCTGA-3' \\ DjAgo 2 reverse: 5'-CATCCATACTGCCTACAACAGC-3' \\ DjpiwiA forward: 5'-GGAGCCATAGGAGAAATCTCATTTG-3' \\ DjpiwiA reverse: $5^{\prime}$-CGCTAATCCAAATCCGGGAAC-3'
}

$D j \beta$-tubulin primers were used as the internal control for normalizing the amount of synthesized cDNA. Primers of DjAgo2 were used for examining DjAgo2 expression. DjpiwiA, a marker gene for neoblasts, were also examined to evaluate the relative amounts of neoblast after depletion of DjAgo2 [18]. The relative mRNA levels of DjAgo2 and DjpiwiA after microinjection or feeding RNAi were determined by using $2^{-\Delta \Delta C t}$ method after RT-qPCR [19].

\section{Results}

\subsection{Microinjection Droplet Volume Calibriation}

Before microinjection can be performed, the injection volume must be calibrated carefully. During the calibration, the glass capillary, loaded with red dye, was immersed in the oil-phase liquid medium. The dye solution was continuously injected into mineral oil to form a droplet for at least one hundred (100) times in the minimum volume. When the red dye was injected into mineral oil, a droplet was formed, departed from the needle tip, and sank downward onto the petri-dish in the bottom. Please note, in some cases, the dye solution stayed attached to the tip, thereby lifting up the glass capillary and detaching the dye solution from the tip to form a droplet.

The radiuses of the droplets were measured under the microscope, as shown in Figure 5, with droplet volumes found by using the equation $\left(\mathrm{v}=4 / 3 \pi r^{3}\right)$, where $\mathrm{v}$ is the volume and $r$ is the radius of the spherical drops. The droplet volume on average was $1.30 \pm 0.24 \mathrm{~nL}$, which is suitable for the experiments since the dose was $50 \mathrm{~nL}$.

The volume of a droplet we measured was larger than its actual value. This was due to the diameter of the droplet becoming a little larger once the droplet adhered to the surface of the petri dish after the droplet precipitated and inhibited onto the surface of the petri dish after the injection. The droplet adhered and "wet" onto the surface, enlarging the diameter during the measurement. In other words, the actual diameter $\left(D_{1}\right)$ of the droplet was smaller than the diameter $\left(D_{2}\right)$ of the droplet that sat on the petri dish (e.g., $\mathrm{D}_{1}<\mathrm{D}_{2}$ ). These diameter measurement difference can be reduced by having a parafilm on petri dish, which would allow the droplets to "dewet". This would allow the droplet to bead up and remain spherical during the measurement. 


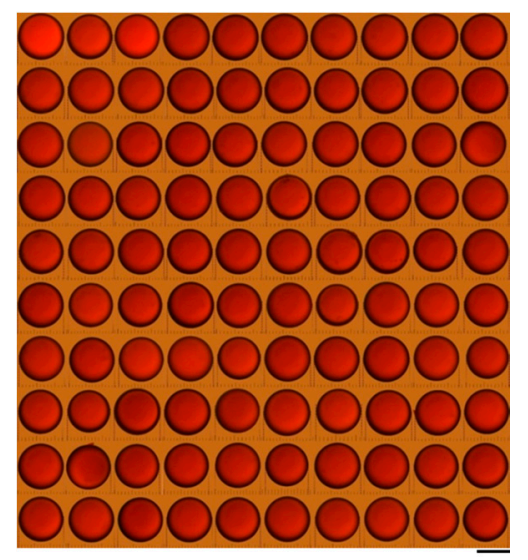

Figure 5. Droplets of red dye in the mineral oil under microscope. The droplet volume on average is $1.30 \pm 0.24 \mathrm{~nL}$. Scale bar $100 \mu \mathrm{m}$.

\subsection{Evaluation on Microinjections}

\subsubsection{Microinjections with Beads}

Microinjections were performed using the fluorescent microbeads for validating the adaptability of our system. Figure 6 showed the images of the planarians, where fluorescent microbeads were injected, under a fluorescent microscope. While there were no fluorescent microbeads in the planarians of the control group, and there were microbeads in two planarians of the experimental group.

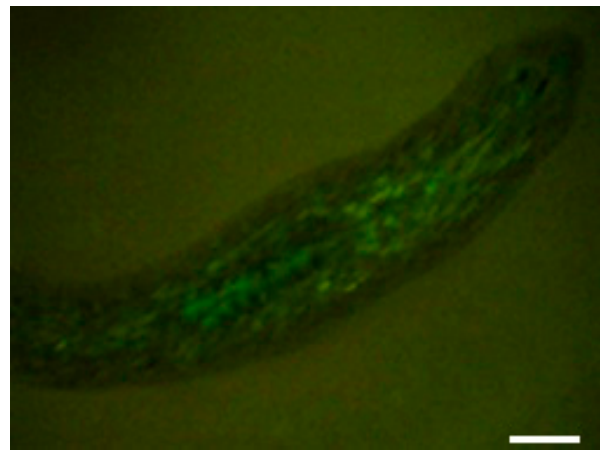

(a)

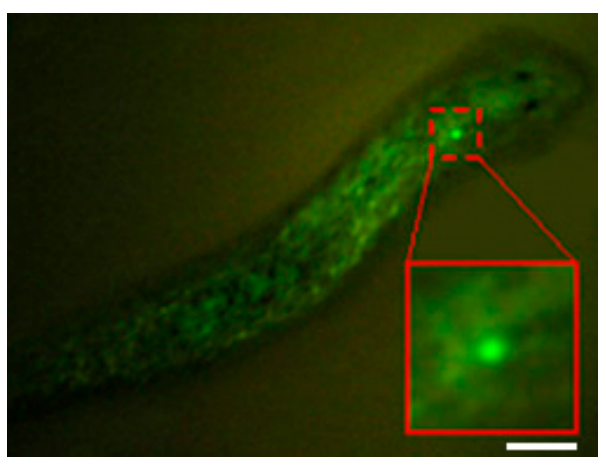

(b)

Figure 6. The fluorescent images of (a) regular planarians without microbeads injected inside (scale bar $500 \mu \mathrm{m}$ ), and (b) a planarian, which had the fluorescent microbeads injected (scale bar $500 \mu \mathrm{m})$.

\subsubsection{Microinjections with DjAgo2 dsRNA}

One of the major advantages of working with planarians is that the expression of endogenous gene can be easily manipulated by microinjection of the droplet solutions containing dsRNA as a reverse genetics tool. Delivery into the cytoplasm is achieved either through direct injection, or through natural endocytosis after ingestion. Successful genetic manipulations are usually followed by quantification of phenotypes to elucidate the genetic mechanisms of development.

The planarians were carefully cultured with their statuses monitored daily since Day 1 after the microinjection. During the days without ingestion, the planarians became smaller, or even died. The appearances (phenotype) of the planarians were recorded every day. On Day 19, right before RNA extraction, the planarians from the control group remained intact after the injections, while the heads of the planarians from the experimental group degenerated significantly. This was consistent with the representative phenotype of DjAgo2-RNAi, which showed the loss of regeneration [20]. 
To quantify the effect of DjAgo2 RNAi, the RT-qPCR was performed to determine the mRNA levels of $D j A g o 2$ in the planarians after Day 19. The results of $D j A g o 2$ dsRNA Injection showed that, in the planarians injected with DjAgo2 dsRNA, the level of DjAgo2 was reduced to $41 \%$ compared with that from the control group (Figure 7a), demonstrating that microinjection RNAi succeeded with a similar knockdown efficiency as feeding RNAi (Figure 7b). Since DjAgo2 was required for the neoblast proliferation and maintenance, the mRNA levels of DjpiwiA (a marker for neoblast) were also examined to confirm whether the neoblasts were also abolished after DjAgo2 RNAi. The RT-qPCR results in Figure 7a showed the level of DjpiwiA in dsAgo2-injected planarians was reduced to 51\% compared with the control group, indicating that DjAgo2 RNAi resulted in $49 \%$ reduction of neoblasts. A similar effect was observed in feeding RNAi (Figure $7 b$ ).

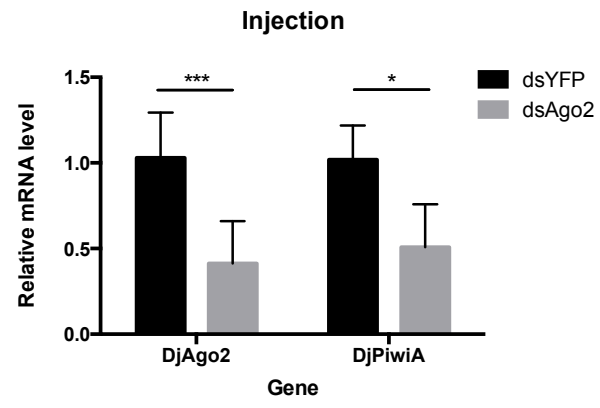

(a)

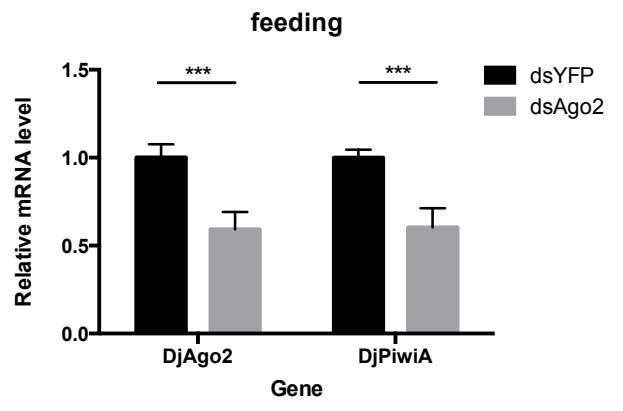

(b)

Figure 7. Significant effects of DjAgo2 RNAi after dsRNA delivery by microinjection. (a) Relative DjAgo2 and DjpiwiA expression levels in the planarians from the control group (dsYFP, $n=8)$ and experimental group (dsAgo2, $n=6$ ) after dsRNA microinjection. ( ${ }^{* * *} p<0.0005$; ${ }^{*} p<0.05$, Student's $t$-test) (b) Relative DjAgo2 and DjpiwiA expression levels in the planarians from the control group (dsYFP, $n=6$ ) and experimental group (dsAgo2, $n=6$ ) after dsRNA feeding. ${ }^{* * *} p<0.0005$, Student's $t$-test).

\section{Discussion}

A gene delivery system using droplet injector and planarian holder was obtained and tested. The microinjector had a minimum droplet injection volume at $1.30 \pm 0.24 \mathrm{~nL}$. Different samples, including microbeads and dsRNA, in the droplets were tested and successfully shown to validate our system. In particular, DjAgo2 dsRNA was successfully delivered into planarians using the microinjector. The result of a reduction of 59\% DjAgo 2 expression validated our microinjection acting as an alternative to ingestion of dsRNA (Figure 7).

One of the main modifications to be made for each neoblast transfection procedure is to control the concentration of injected samples. For instance, a high concentration of dsRNA may cause non-specific toxicity. These tests usually are empirically done to determine the optimal concentration; typically dsRNA concentrations between 200 and $500 \mu \mathrm{M}$ to effectively knock-down gene without causing nonspecific defects. The precise size of the needle opening is not crucial. Within a range of needle sizes, injection pressure and injection time can be adjusted to produce a correct volume for the injection droplet. In our experiments, capillaries with a variety of needle opening sizes (e.g., I.D. $=10 \sim 30 \mu \mathrm{m}$ ) have been tested to determine the optimal sizes for higher efficiency and successful rates in neoblast transplantations.

Our planarian holder, with a feedback control support temperatures of $12.00 \pm 0.02{ }^{\circ} \mathrm{C}$. The temperature fluctuation on the platform in the steady state was greatly reduced, thereby minimizing steady-state error. Compared to a conventional approach, which usually uses ice to freeze the planarian, our temperature-controlled holder provides a "dry" environment in which to fix the planarian, thereby simplifying the microinjection easy while keeping the injection dose constant. 
Further, one possible application of our injection system is to perform neoblasts transfection from a normal planarian (planarian A) to a $\gamma$-irradiation exposed planarian (planarian B), which has lost its ability for regeneration, then observe how the neoblasts can be regenerated in planarian $B$ to form a new planarian, which is identical to planarian A. The mechanism of planarian regeneration would be understood.

Finally, our microinjector can be applicable to other creatures like insects, zebra fishes, or even mice with low volume at the dose of $1.3 \mathrm{~nL}$. Single cell (e.g., embryo) injection requires nanoliter-scale injection to prevent the rupture of the cell membrane due to high tension [8,9]. Microinjections of dsRNAs for other planarian genes at similar injection volume (e.g., 1.5 100 nL) are also possible to be performed in the future, in particular, for the genes whose functions are related to starvation conditions.

Author Contributions: Conceptualization, M.L. and C.-Y.C.; Methodology, M.L.; Validation, M.L. and C.-Y.C.; Formal Analysis, M.L.; Investigation, M.L. and C.-Y.C.; Resources, C.C. and Y.-W.L.; Data Curation, M.L., J.-J.S. and C.-Y.C.; Writing-Original Draft Preparation, M.L.; Writing-Review \& Editing, Y.-W.L.; Supervision, C.-Y.C. and Y.-W.L.; Project Administration, Y.-W.L.; Funding Acquisition, Y.-W.L.

Funding: This research was funded by Ministry of Science and Technology grant number MOST 106-2221-E-002-144 and MOST 107-2221-E-002-130-MY3.

Acknowledgments: Authors gratefully acknowledge P.-Y. Chiou at University of California, Los Angeles for initial discussion about the project, and T. Kirk for manuscript editing.

Conflicts of Interest: The authors declare no conflict of interest.

\section{References}

1. Gimble, J.M.; Katz, A.J.; Bunnell, B.A. Adipose-derived stem cells for regenerative medicine. Circ. Res. 2007, 100, 1249-1260. [CrossRef] [PubMed]

2. Mason, C.; Manzotti, E. Regenerative medicine cell therapies: Numbers of units manufactured and patients treated between 1988 and 2010. Regener. Med. 2010, 5, 307-313. [CrossRef] [PubMed]

3. Reddien, P.W.; Alvarado, A.S. Fundamentals of planarian regeneration. Annu. Rev. Cell Dev. Biol. 2004, 20, 725-757. [CrossRef] [PubMed]

4. Pedersen, K.J. Cytological studies on the planarian neoblast. Cell Tissue Res. 1959, 50, 799-817. [CrossRef]

5. Baguna, J. The planarian neoblast: The rambling history of its origin and some current black boxes. Int. J. Dev. Biol. 2012, 56, 19-37. [CrossRef] [PubMed]

6. Rouhana, L.; Weiss, J.A.; Forsthoefel, D.J.; Lee, H.; King, R.S.; Inoue, T.; Shibata, N.; Agata, K.; Newmark, P.A. RNA interference by feeding in vitro-synthesized double-stranded RNA to planarians: Methodology and dynamics. Dev. Dyn. 2013, 242, 718-730. [CrossRef] [PubMed]

7. Newmark, P.A.; Reddien, P.W.; Cebrià, F.; Alvarado, A.S. Ingestion of bacterially expressed double-stranded RNA inhibits gene expression in planarians. Proc. Natl. Acad. Sci. USA 2003, 100, 11861-11865. [CrossRef] [PubMed]

8. Feramisco, J.; Perona, R.; Lacal, J.C. Needle Microinjection: A Brief History. In Microinjection; Lacal, J.C., Feramisco, J., Perona, R., Eds.; Birkhäuser: Basel, Switzerland, 1999; pp. 9-15.

9. Capecchi, M.R. High efficiency transformation by direct microinjection of DNA into cultured mammalian cells. Cell 1980, 22, 479-488. [CrossRef]

10. Li, S.; Zheng, D.; Li, N.; Wang, X.; Liu, Y.; Sun, M.; Zhao, X. Size-adjustable microdroplets generation based on microinjection. Micromachines 2017, 8, 88. [CrossRef]

11. Rosen, J.N.; Sweeney, M.F.; Mably, J.D. Microinjection of zebrafish embryos to analyze gene function. J. Visual. Exp. 2009, 25. [CrossRef] [PubMed]

12. Gordon, J.W.; Scangos, G.A.; Plotkin, D.J.; Barbosa, J.A.; Ruddle, F.H. Genetic transformation of mouse embryos by microinjection of purified DNA. Proc. Natl. Acad. Sci. USA 1980, 77, 7380-7384. [CrossRef] [PubMed]

13. Noori, A.; Selvaganapathy, P.R.; Wilson, J. Microinjection in a microfluidic format using flexible and compliant channels and electroosmotic dosage control. Lab Chip 2009, 9, 3202-3211. [CrossRef] [PubMed]

14. Ansorge, W.; Pepperkok, R. Performance of an automated system for capillary microinjection into living cells. J. Biochem. Biophys. Methods 1988, 16, 283-292. [CrossRef] 
15. Song, P.; Dong, X.; Liu, X. A microfluidic device for automated, high-speed microinjection of Caenorhabditis elegans. Biomicrofluidics 2016, 10, 011912. [CrossRef] [PubMed]

16. Zhao, X.; Xu, F.; Tang, L.; Du, W.; Feng, X.; Liu, B.-F. Microfluidic chip-based C. elegans microinjection system for investigating cell-cell communication in vivo. Biosens. Bioelectron. 2013, 50, 28-34. [CrossRef] [PubMed]

17. Shibata, N.; Agata, K. RNA interference in planarians: Feeding and injection of synthetic dsRNA. Methods Mol. Biol. 2018, 1774, 455-466. [PubMed]

18. Rossi, L.; Salvetti, A.; Lena, A.; Batistoni, R.; Deri, P.; Pugliesi, C.; Loreti, E.; Gremigni, V. DjPiwi-1, a member of the PAZ-Piwi gene family, defines a subpopulation of planarian stem cells. Dev. Genes Evol. 2006, $216,335$. [CrossRef] [PubMed]

19. Livak, K.J.; Schmittgen, T.D. Analysis of relative gene expression data using real-time quantitative PCR and the $2^{-\Delta \Delta C t}$ Method. Methods 2001, 25, 402-408. [CrossRef] [PubMed]

20. Li, Y.-Q.; Zeng, A.; Han, X.-S.; Wang, C.; Li, G.; Zhang, Z.-C.; Wang, J.-Y.; Qin, Y.-W.; Jing, Q. Argonaute-2 regulates the proliferation of adult stem cells in planarian. Cell Res. 2012, 22, 275. [CrossRef]

(C) 2018 by the authors. Licensee MDPI, Basel, Switzerland. This article is an open access article distributed under the terms and conditions of the Creative Commons Attribution (CC BY) license (http:/ / creativecommons.org/licenses/by/4.0/). 